\title{
Alveolar Osteitis Following Tooth Extraction: Systematic Review and Google Trends Analysis Instigated by the First Upper Premolar Case Reported from Iraq
}

\author{
Ahmed Al-Imam ${ }^{1,2,3,4} \&$ Amer Al-Khazraji ${ }^{5,6,7}$ \\ ${ }^{1}$ Department of Anatomy and Cellular Biology, College of Medicine, University of Baghdad, Iraq \\ ${ }^{2}$ CERVO Brain Research Centre, Faculty of Medicine, University of Laval, Canada \\ ${ }^{3}$ The Canadian Association for Neuroscience, Iraq \\ ${ }^{4}$ The Japanese Association of Anatomists, Iraq \\ ${ }^{5}$ Consultant of Oral and Maxillofacial Surgery, Iraq \\ ${ }^{6}$ Scientific Advisor of the Iraqi Dental Association, Iraq \\ ${ }^{7}$ Lecturer and Supervisor at the British Academy of Implant and Restorative Dentistry, Iraq \\ Correspondence: Ahmed Al-Imam, Department of Anatomy and Cellular Biology, College of Medicine, \\ University of Baghdad, Bab Al-Mo'adham, 10053, Baghdad, Iraq. E-mail: tesla1452@gmail.com, \\ ahmed.al-imam.1@ulaval.ca
}

Received: December 25, 2018

Accepted: January 11, 2019 Online Published: February 14, 2019

doi:10.5539/mas.v13n3p1

URL: https://doi.org/10.5539/mas.v13n3p1

\begin{abstract}
Introduction

Alveolar osteitis is a painful condition that may occur following permanent teeth extraction due to the failure of formation of a blood clot or its dislodgement before the complete healing of the wound. We aim to provide a systematic review and trends analytic on the epidemiology and the digital epidemiology as well as the management of alveolar osteitis and to seek any available data in connection with alveolar osteitis following upper premolar tooth extraction.
\end{abstract}

\section{Methods}

This study represents a combinatory of literature review, analytics of Google Trends, and the first documented case from Iraq of alveolar osteitis following extraction of the maxillary first premolar. Three literature databases were explored, using Boolean operators, including NCBI-PubMed, Elsevier, and the Cochrane Library. Google Trends database was examined to assess the digital epidemiology.

\section{Results}

The total number of hits was 54417. There was an overall deficit of literature concerning the condition in connection with the extraction of maxillary premolars. The digital epidemiology was limited to twenty-two countries including three countries from the Middle East accounting for $13.63 \%$ of the total geographic mapping while Iraq was absent.

\section{Conclusion}

Our exceptional case report instigated a systematic analytic of a trends database and the literature. The analysis confirmed the inadequacy of studies from the Middle East. Future studies should deploy the use of machine learning algorithms for a rigour statistical inference based on data from online and offline big data repositories of public health records.

Keywords: dry socket, alveolar osteitis, evidence-based medicine, google trends, Arabs 


\section{Introduction}

Alveolar osteitis is one of the most frequent postoperative complications following permanent teeth extraction. This term exists in the literature since 1896, when Crawford first described it in 1896 (Crawford, 1896; Blum, 2002). Since then, several other names were coined including alveolar osteitis (AO), localised osteitis, alveolalgia postoperative alveolitis, fibrinolytic alveolitis, alveolitis sicca dolorosa, septic socket, necrotic socket, and localised osteomyelitis (Crawford, 1896; Blum, 2002; Abraham, Azari, Westcott, \& Stavropoulos, 2016). Evidence-Based Medicine (EBM) is a systematic approach of critical importance to medical practice intended to optimise decision-making by emphasising the value and use of evidence from well-designed research studies (Greenhalgh, 2014; Hoffmann, Montori, \& Del Mar, 2014). One of the most rigour EBM- related institutes is the Centre for Evidence-Based Medicine which was established in Oxford in 1995 (Phillips et al., 2011; Oxford Centre for Evidence-Based Medicine, 2016). It aims to promote evidence-based health care and up-to-date decision-making in health care around the world. In parallel to EBM, the critical appraisal of scientific literature embodies a vital skill to be mastered by academic medical professionals as well as others who are involved in clinical practice (Shea et al., 2017; Better Value Healthcare Ltd, 2018). There is an abundance of medical and paramedical databases of published literature. However, there are also some essential unpublished studies indexed in the grey literature including Open Grey database (Adams, Smart, \& Huff, 2017; Giannini \& Molino, 2018). Nevertheless, literature databases represent a more valid reference material as it is peer-reviewed as in case of publications indexed via the National Library of Medicine (NLM) and the PubMed Central archive of biomedical research (Wheeler, 2006). Fewer databases of clinical trials, though of higher evidence, exists and even fewer databases are dedicated for controlled and randomised controlled clinical trials (RCTs) conducted on humans as in the case of the Cochrane Library and its Central Register of Controlled Trials (Cochrane, 2018).

Databases of trends as well as repositories of public health record also represent an invaluable source of big data that can be used for a wide-scale global statistical inference (Lent, Agrawal, \& Srikant, 1997; Nuti et al., 2014). Those databases include real (offline) databases and virtual (online) databases existing on the surface web. On the other hand, the invisible web (deep web) which is unknown to many readers can be an immense source in connection to issues related to public health including studies related to addictions and substance (ab)use, the electronic commerce of illicit chemical substances and controlled drugs, and even in relation to the digital epidemiology of some diseases (Al-Imam, 2017; Al-Imam \& AbdulMajeed, 2017). Our study will constitute a comprehensive review of the literature with particular attention to clinical trials of therapeutic modalities and analytics of Google Trends databases. Concepts of real-time and predictive analytics, using principles of machine learning, will be a cornerstone in future epidemiological studies and on a massive scale (Nasrabadi, 2007; Hay, George, Moyes, \& Brownstein, 2013; Al-Imam, 2019; Al-Imam, Khalid, Al-Hadithi, \& Kaouche, 2019). These concepts are not limited to pattern recognition, neural networks, and automated analytics of inference of big data that are vital to be implemented across the databases above to extrapolate rigour level-of-evidence (Gašević, Dawson, \& Siemens, 2015; Poulin, Thompson, \& Bryan, 2016; Steele, Chandler, \& Reddy, 2016; Witten, Frank, Hall, \& Pal, 2016).

\section{Materials and Methods}

Three databases of literature were explored on the $26^{\text {th }}$ and the $27^{\text {th }}$ of July 2018 , including PubMed-NCBI, Elsevier, and the Cochrane Library which hosts the Central Register of Controlled Trials known as CENTRAL (Table 1). Keywords based on Medical Subject Headings (MeSH) were used in solitary and in various combinations using Boolean operators (AND, OR, NOT) to retrieve the most relevant hits (manuscripts) concerning the topic of our research. Bibliographic materials of interest were assessed and appraised for validity and rigorousness via critical appraisal tools (Better Value Healthcare Ltd, 2018). Studies that successfully passed the critical appraisal were considered as potential reference materials. Those studies were conducted exclusively in humans and written in the English language. Priority was given to recently published literature within the past $5-10$ years.

Online databases including Google Trends and other trends databases can be consulted to assess the extent of digital epidemiology and the relevant geographic mapping of this rare medical entity and based on the interest of users of the surface web on the topic of interest (Lent et al., 1997; Nuti et al., 2014; Al-Imam, 2017; Al-Imam \& AbdulMajeed, 2017). Geographic mapping can also be used to evaluate the fractional contribution of the Middle East and Arabic countries to the global map. We explored Google Trends for the past five years via two keywords "dry socket" and "alveolar osteitis". Statistical analysis was conducted via SPSS version 24 (SPSS v.24, 64-bits Operating System). Our case report represents the first incidence from Baghdad, the capital city of Iraq. It occurred during July 2018. The level-of-evidence of this hybrid-study analytic ranks as level-3 within the hierarchy of evidence as proposed by the Oxford Centre for Evidence-based Medicine (CEBM) in 2016 (Phillips 
et al., 2011; Oxford Centre for Evidence-Based Medicine, 2016).

Table 1. Literature Databases and the Number of Hits based on Keywords Search

\begin{tabular}{|c|c|c|c|c|}
\hline \multirow[b]{2}{*}{ Keyword (MeSH terms) } & \multicolumn{4}{|c|}{ Number of Hits per Database } \\
\hline & PubMed-NCBI & The Cochrane Library & Elsevier & Total \\
\hline dry socket & 91 & 3 & 8 & 102 \\
\hline alveolar osteitis & 125 & 3 & 9 & 137 \\
\hline "dry socket" & 80 & 3 & 8 & 91 \\
\hline "alveolar osteitis" & 58 & 3 & 9 & 70 \\
\hline "dry socket" OR "alveolar osteitis" & 107 & 4 & 0 & 111 \\
\hline $\begin{array}{l}\text { "maxillary premolar" OR "upper } \\
\text { premolar" }\end{array}$ & 115 & 160 & 0 & 275 \\
\hline "extraction" & 49431 & 1008 & 2119 & 52558 \\
\hline "tooth" AND extract* & 913 & 156 & 1 & 1070 \\
\hline $\begin{array}{l}\text { (“dry socket" OR "alveolar osteitis") } \\
\text { AND ("maxillary premolar" OR "upper } \\
\text { premolar") }\end{array}$ & 0 & 1 & 0 & 1 \\
\hline $\begin{array}{l}\text { (“dry socket" OR “alveolar osteitis") } \\
\text { AND ("maxillary premolar" OR "upper } \\
\text { premolar") AND extract* }\end{array}$ & 0 & 1 & 0 & 1 \\
\hline $\begin{array}{l}\text { (“dry socket" OR "alveolar osteitis") } \\
\text { AND (“maxillary premolar" OR "upper } \\
\text { premolar") AND ("tooth" AND extract*) }\end{array}$ & 0 & 1 & 0 & 1 \\
\hline $\begin{array}{l}\text { (“dry socket" OR “alveolar osteitis") } \\
\text { AND ("maxillary premolar" OR "upper } \\
\text { premolar") AND extract* AND "middle } \\
\text { east” }\end{array}$ & 0 & 0 & 0 & 0 \\
\hline $\begin{array}{l}\text { (“dry socket” OR “alveolar osteitis") } \\
\text { AND ("maxillary premolar" OR "upper } \\
\text { premolar") AND extract* AND iraq }\end{array}$ & 0 & 0 & 0 & 0 \\
\hline $\begin{array}{l}\text { (“dry socket" OR “alveolar osteitis") } \\
\text { AND (“maxillary premolar" OR "upper } \\
\text { premolar") AND extract* AND (arab OR } \\
\text { "middle east") }\end{array}$ & 0 & 0 & 0 & 0 \\
\hline $\begin{array}{l}\text { (“dry socket” OR “alveolar osteitis”) } \\
\text { AND (“maxillary premolar” OR "upper } \\
\text { premolar") AND extract* AND (iraq OR } \\
\text { arab) }\end{array}$ & 0 & 0 & 0 & 0 \\
\hline $\begin{array}{l}\text { (“dry socket" OR "alveolar osteitis") } \\
\text { AND (“maxillary premolar" OR "upper } \\
\text { premolar") AND extract* AND (iraq OR } \\
\text { arab OR “middle east”) AND report* }\end{array}$ & 0 & 0 & 0 & 0 \\
\hline Total Number of Hits & 50920 & 1343 & 2154 & 54417 \\
\hline
\end{tabular}

\section{Results}

\subsection{Databases of Literature}

Based on a review of the literature databases, the summative number of hits was 54417 distributed as 50920 (PubMed-NCBI), 1343 (the Cochrane Library), and 2154 (Elsevier). Two combinations of Keywords generated the most valid hits including ["dry socket" OR "alveolar osteitis"] as well as ["dry socket" OR "alveolar osteitis") AND ("maxillary premolar" OR "upper premolar") AND ("tooth" AND extract*]. Unfortunately, more advanced combinations of keywords yielded absolutely no hits which indicate the lack of published literature in connection with the topic of dry socket and alveolar osteitis following the extraction of maxillary premolars. Besides, the body of literature has a complete absence of research attempts originating from the Middle East, specifically 
from Iraq. Following the application of inclusion criteria, the total number of reference materials used for the citation and the purpose of a literature review reached fifty-five. Following the elimination of duplicate articles, the inclusion criteria were based on the selection of papers written in the English language for studies and experimentations on human as well as using animal models, and successfully passing the critical appraisal using CASP appraisal tool (Better Value Healthcare Ltd, 2018).

\subsection{Google Trends Database}

Concerning the analytics via Google Trends databases, the contributing countries were twenty-two (22) included the United States, Ireland, Canada, New Zealand, Australia, United Kingdom, Singapore, South Africa, Philippines, United Arab Emirates (UAE), Norway, Denmark, Malaysia, Sweden, Pakistan, Kingdom of Saudi Arabia (KSA), India, Egypt, Thailand, Indonesia, Netherlands, and Germany. Digital epidemiology was lacking for Iraq, while countries from the Middle East were limited to the UAE, KSA, and Egypt which accounts for $13.63 \%$ of the total geographic mapping (Figure 1). Hypothesis testing and parametric statistical inference based on Student's t-test, confirmed the existence of a statistically significant difference in the percentile representation of the interest of surface web users on the topic and based on the two keywords "dry socket" and "alveolar osteitis" $(99.86+/-0.14$ versus $0.23+/-0.15$, p-value $<0.001)$. This finding goes in line with the critical analysis of the published literature and further confirms the inadequacy of the published literature concerning this rare dentistry phenomenon. Google Trends when combined with machine learning and data crunching algorithms can extrapolate real-time and predictive analytics of the trends (Nasrabadi, 2007; Hay et al., 2013; Gašević et al., 2015; Poulin et al., 2016; Steele et al., 2016; Witten et al., 2016; Al-Imam, 2019; Al-Imam et al., 2019).
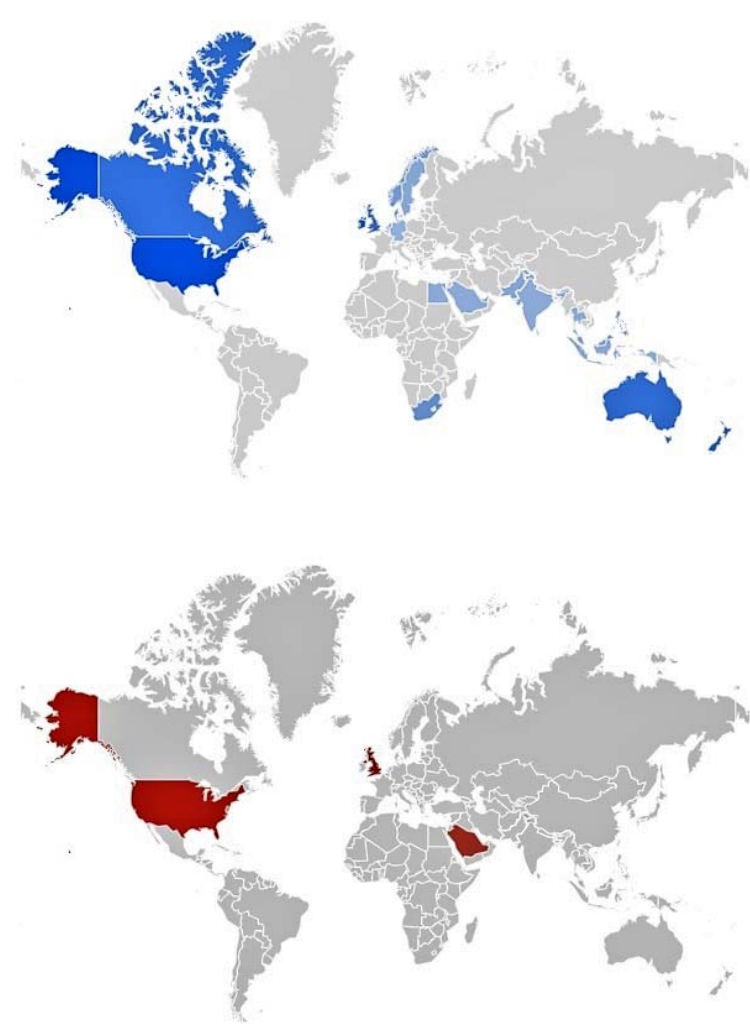

Figure 1. Geographic Mapping via Google Trends: "Dry Socket” Keyword (colour-coded blue) and "Alveolar Osteitis" (colour-coded red)

\section{Discussion}

\subsection{Discussion of Literature}

\subsubsection{Epidemiology, Demographics, and Risk Factors}

For routine dental extractions, the incidence of $\mathrm{AO}$ has been reported in the range $0.5 \%$ to $5 \%$, while the dry socket is the most common complication following third molar (wisdom tooth) extraction. However, the incidence of $\mathrm{AO}$ after removal of mandibular third molars varies from $1 \%$ to $37.5 \%$ (Crawford, 1896; Blum, 
2002; Abraham, et al., 2016). It has been well documented that surgical extractions result in about ten times higher incidence of AO. Other risk factors include developing a dry socket in the past, drinking fluids with a straw and vigorous mouth rinsing following tooth extraction, smoking and tobacco chewing, use of oral contraceptives and hormonal replacement therapy, and poor oral hygiene as well as infection of the gums and teeth (Crawford, 1896; de Wyels, 1998; Bosco, Oliveira, Bosco, Schweitzer, \& Jardim Júnior, 2008; Krakowiak, 2011; Sharif, Dawoud, Tsichlaki, \& Yates, 2014). Besides, it has been found the tooth extraction during the menses decreases the risk of developing a dry socket in females.

\subsubsection{Studies in Non-Human Species}

Two studies were found in animals, a case report and a trial (de Wyels, 1998; Bosco, et al., 2008). De Wyels reported a case of AO in an eight-year-old retriever dog following the extraction of a maxillary left first molar tooth. The alveolus was managed with curettage followed by a medical regimen of cefalexin and prednisolone (de Wyels, 1998). Bosco and colleagues conducted an experimental study on forty Wistar rats assigned to four groups to compare various treatment modalities following tooth extraction including incisors and canines. The use of tetracycline reduced the occurrence of $\mathrm{AO}$ and caused significant changes in the microbiota of the surgical sites, decreasing the anaerobes and increasing the population of tetracycline-resistant and multi-resistant microorganisms (Bosco, et al., 2008).

\subsubsection{Case Reports, Case Series, and Reviews}

Alveolar osteitis is a frustrating and common post-operative complication following exodontia (Krakowiak, 2011; Sharif et al., 2014; Cho, Lynham, \& Hsu, 2017). Cho, Lynham, and Hsu concluded that paracetamol and ibuprofen are efficacious in managing postoperative pain, while antibiotics and corticosteroids should only be used in selected cases. The benefits of cryotherapy, postoperative irrigation and ozone gel are yet to be established (Cho et al., 2017). In 2017, Fernandes and Hatton described a novel technique for the prevention of $\mathrm{AO}$ via the administration of doxycycline dispersed in a local anaesthetic solution while using a Gelfoam carrier (Fernandes \& Hatton, 2017). In 2011, an audit carried out in the Dublin Dental School and Hospital, showed that a wide range of treatments are used with success for the treatment of alveolar osteitis including rinsing of the socket with chlorhexidine ( $74 \%$ ) or saline (26\%), placement of a non-resorbable obtundent dressing $(56 \%)$, and home-based rinsing of the socket with chlorhexidine (44\%) (Bowe, 2011).

\subsubsection{Experimental Clinical Trials}

Several measures are used to reduce the incidence of AO including warm saline mouth rinse, the use of prophylactic antibiotics, chlorhexidine mouthwash, and platelet-rich fibrin (Eshghpour, Dastmalchi, Nekooei, \& Nejat, 2014; Osunde, 2015; Xue et al., 2015; Halabi, Escobar, Alvarado, Martinez, \& Muñoz, 2018; Unsal \& Erbasar, 2018). However, Xue and co-authors confirmed that Prophylactic amoxicillin (or clindamycin) is not effective for the prevention or reduction of postoperative inflammatory complications after the removal of impacted mandibular third molars in the Chinese population (Xue et al., 2015). On the other hand, Tek and co-workers found that Ankaferd Blood Stopper (ABS) can be used safely for haemostasis following an impacted mandibular third molar surgery without potentially increasing the risk of AO formation (Tek et al., 2014). Very recently, Halabi and others, verified that $0.12 \%$ chlorhexidine mouthwash is safe and effective in reducing the incidence of AO in high-risk patients (Halabi et al., 2018). Unsal and colleagues as well as Eshghpour and co-workers verified in their experimental clinical trials that platelet-rich fibrin (PRF) significantly reduced the AO incidence, especially following mandibular third molar surgery, among smokers, and it also had a positive effect on postoperative pain levels although it did not affect periodontal healing (Eshghpour \& Dastmalchi, 2014; Unsal \& Erbasar, 2018).

Concerning the management of AO, several trials emphasised the importance of bio-adhesive chlorhexidine gel, hyaluronic acid and aminocaproic acid, and the potentials of low-level laser therapy (LLLT) (Abu-Mostafa, Alqahtani, Abu-Hasna, Alhokail, \& Aladsani, 2015; Eshghpour, Ahrari, Najjarkar, \& Khajavi, 2015; Freudenthal, Sternudd, Jansson, \& Wannfors, 2015; Haraji \& Rakhshan , 2015; Jesudasan, Wahab, \& Sekhar, 2015; Rubio-Palau et al., 2015; Dubovina et al., 2016). Five well-designed clinical trials extensively investigated the therapeutic effects of intra-alveolar bio-adhesive chlorhexidine gel $(0.2 \%)$ in comparison with other therapeutic modalities as well as a placebo (Abu-Mostafa et al., 2015; Freudenthal et al., 2015; Haraji \& Rakhshan, 2015; Jesudasan, et al., 2015; Rubio-Palau et al., 2015). Jesudasan validated that the eugenol was the better treatment modality as it was successful in reducing pain, inflammation, infection, and promoting a better wound healing (Jesudasan et al., 2015). Freudenthal could not verify that the application of chlorhexidine gel improves healing after the removal of impacted third molars (Freudenthal et al., 2015). Rubio-Palau and colleague verified a $22 \%$ reduction of the incidence of alveolar osteitis with the application of chlorhexidine gel compared to placebo 
although it was not statistically significant (Rubio-Palau et al., 2015). However, the authors favoured the use of the gel when compared to mouth rinses as chlorhexidine did not show any adverse reactions and complications. Further, Abu-Mostafa and coworkers carried out an evaluation of the molar extraction sockets, he confirmed that patients who received direct $0.2 \%$ Chlorohexidine gel did not show any significant difference when compared with $0.12 \%$ Chlorohexidine rinse and in connection with reducing the incidence of AO (Abu-Mostafa et al., 2015). Nevertheless, in 2015 Haraji confirmed that chlorhexidine gel regardless of its therapeutic effects can reduce the pain effectively in patients although a few confounding variables, including age and sex as well as the smoking status, require verification via multivariate analytics (Haraji \& Rakhshan, 2015).

Other novel modalities of treatment exist. In 2016, Dubovina and co-workers confirmed that the use of Hyaluronic acid, with or without aminocaproic acid, significantly reduces pain sensation and can be successfully used in treating AO (Dubovina et al., 2016). One year earlier, Eshghpour found that low-level laser therapy (LLLT) also displayed good results in treating alveolar osteitis and was considered as a potential alternative to alvogyl in managing AO (Eshghpour et al., 2015). Concerning surgical procedures, Koyuncu and Çetingül confirmed that using the modified triangular flap for an impacted mandibular third molar surgery, had the advantage of less postoperative swelling and pain but had the disadvantage of developing a dry socket (Koyuncu \& Çetingül, 2013). Elo and co-researchers found, via their split-mouth clinical trial, that one flap technique known as the mesial papilla-sparing marginal incision with distobuccal release with double-pass single-layered primary closure (MPMI-2 X), was a reliable technique to reduce post-operative complications including AO and wound dehiscence following a mandibular third molar surgery (Elo, Sun, Dong, Tandon, \& Singh, 2016).

\subsubsection{Systematic Reviews and Metanalytic Studies}

Several risk factors are known to be correlated with the development of a dry socket, and there have been few studies about the oral contraceptives (OC) as a risk factor. In their metanalytic study in 2016, Bienek and Filliben discovered that the use of OC significantly increased the risk of dry socket development by nearly two folds (Bienek \& Filliben, 2016). Taberner-Vallverdú and colleagues concluded that there is a heterogeneity of interventions, and these were not limited to curettage and irrigation, antiseptics, LLLT, and zinc oxide eugenol or plasma rich in growth factors (Taberner-Vallverdú, Nazir, Sánchez-Garcés, \& Gay-Escoda, 2015). Marcussen found that a single preoperative oral dose of 2 grams of amoxicillin for lower third molar osteotomy surgical extraction, significantly decreased the incidence of surgical site infection while a single dose of 0.8 gram of penicillin- $\mathrm{V}$ significantly reduced the incidence of alveolar osteitis (Marcussen, Laulund, Jørgensen, \& Pinholt, 2016).

Chlorhexidine was the centre of several metanalytic studies and systematic reviews conducted from 2012 to 2016. In 2012, Yengopal and Mickenautsch analytics confirmed that Chlorhexidine did not cause higher adverse reactions in comparison with placebo although the coauthors stressed the importance of conducting further high-quality RCTs (Yengopal \& Mickenautsch, 2012). During the same year, Daly and coresearchers investigated the local interventions for the management of alveolar osteitis as a complication for third molar extraction, they concluded that there is some evidence that rinsing with chlorhexidine $(0.12 \%$ and $0.2 \%)$ or placing chlorhexidine gel $(0.2 \%)$ in the sockets of extracted teeth, provides a benefit in preventing dry socket (Daly, Sharif, Newton, Jones, \& Worthington, 2012). However, there was insufficient evidence to determine the effects of any of the interventions to treat alveolar osteitis although they have also recommended that all members of the dental community prescribing chlorhexidine products to be aware of the minor and serious adverse side effects including potential allergies. Later in 2017, Sánchez and colleagues concluded that the use of chlorhexidine, in any formulation-concentration or regimen, is effective in preventing $\mathrm{AO}$ in patients with third molar extraction although the chlorhexidine gel was moderately more efficacious than the rinse formulation (Sánchez, Andrés, \& Calvo, 2017). Two other metanalytic studies reached a similar conclusion on the higher efficacy of the use of chlorhexidine gel when compared to a placebo and following mandibular third molar extraction (Teshome, 2017; Zhou, Hu, Liu, Yang, \& Song, 2017).

\subsection{Case Presentation}

The patient is thirty-five years old male of the Arab ethnicity from Iraq. He is a non-smoker and healthy apart from an asymptomatic $8 \mathrm{~mm}$ hemangioma that was discovered accidentally, via abdominal ultrasonography, within the hepatic tissue of his liver since 2014. He was also diagnosed with a lumbar spine problem including a torn multifidus muscle although his body mass index (BMI) is acceptable (24.2) and within the normal range at (18.5-24.9). The patient does not have any significant history concerning the musculoskeletal system including fractures, arthritis, or any other pathologies. The patient was complaining of bad dental health due to crowded teeth, particularly in his lower jaw (mandible), that lead to dental carries affecting nearly $50 \%$ of all his teeth for 
which he consulted several dentists and on multiple occasions over the last decade of his life (Figure 2 and 3 ). The dentists managed his problem with fillings including root canal filling for five of his teeth including molars and premolars both maxillary and mandibular. The patient had complications for two of those teeth, the maxillary first premolars (upper fourth), mainly a periapical abscess for which tooth extraction of the left upper fourth tooth was mandatory.

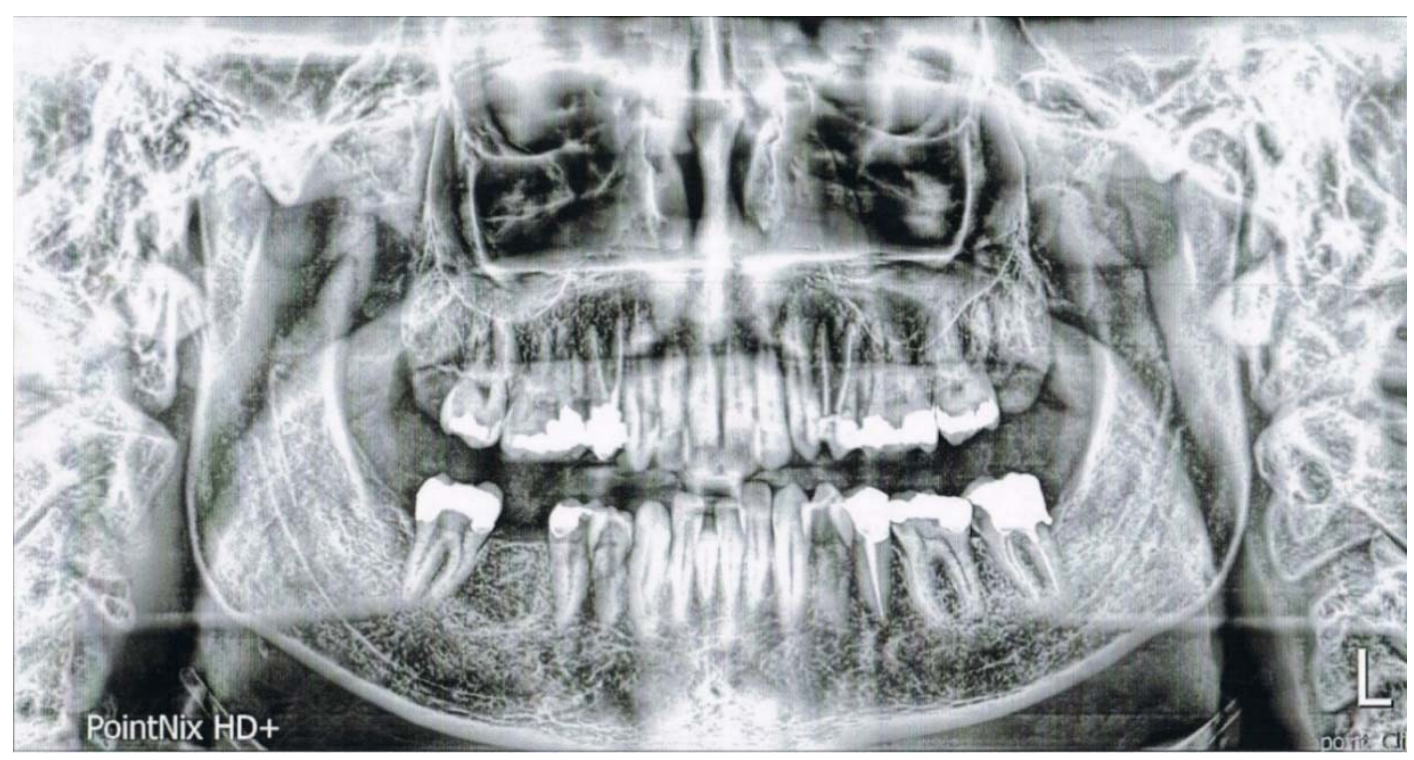

Figure 2. Orthopantomogram (OPG): Panoramic View before the Extraction of the Maxillary Premolars.

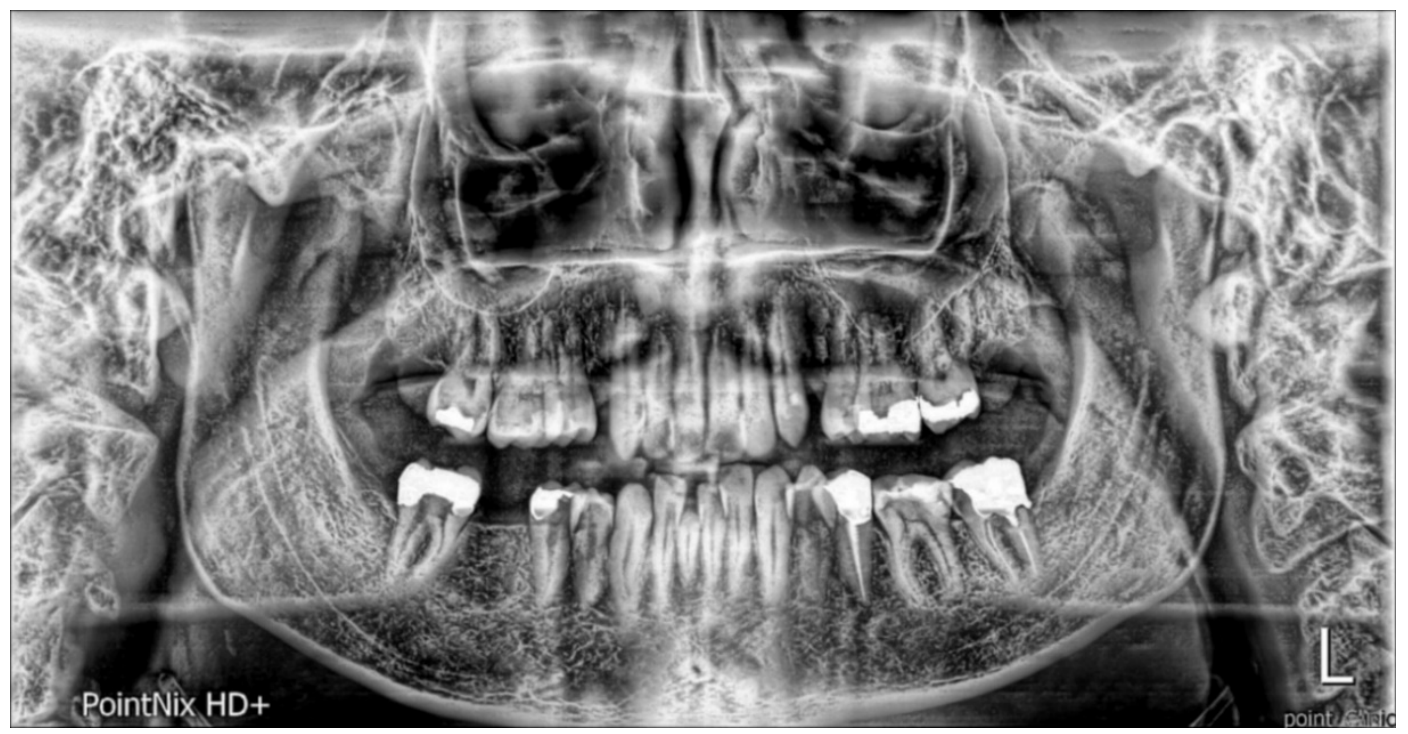

Figure 3. Orthopantomogram (OPG): Panoramic View after the Extraction of the Maxillary Premolars

Based on the complicated history of dental health, he decided to make a consultation concerning his original tooth crowding problem, and the dentist advised him to go with an orthodontic approach. Subsequently, the patient had a second extraction of the contralateral premolar as part of his orthodontic management plan. However, the procedure was traumatic and took nearly 30 minutes. The tooth was broken into halves at the level of the neck. The healing of the alveolar socket was delayed, and the unfortunate patient developed an unanticipated dry socket and a subsequent alveolar osteitis within three days from the distressing extraction operation. As a result, he re-consulted the doctor for the chronic pain who diagnosed it as an unusual case of alveolar osteitis that was managed with non-steroidal painkillers, antibiotics and antimicrobials in the form of Rodogyl oral tablets for ten days. The dentists cleansed and irrigated the alveolar wound, and surgically debrided the necrotised bone sequestra, and put a medicated foam pack impregnated with a conventional antiseptic 
secured deep within the alveolar socket for four days. On the next dentistry visit, the foam was removed, and the wound was partially closed by suturing it with three stitches (Figure 4).
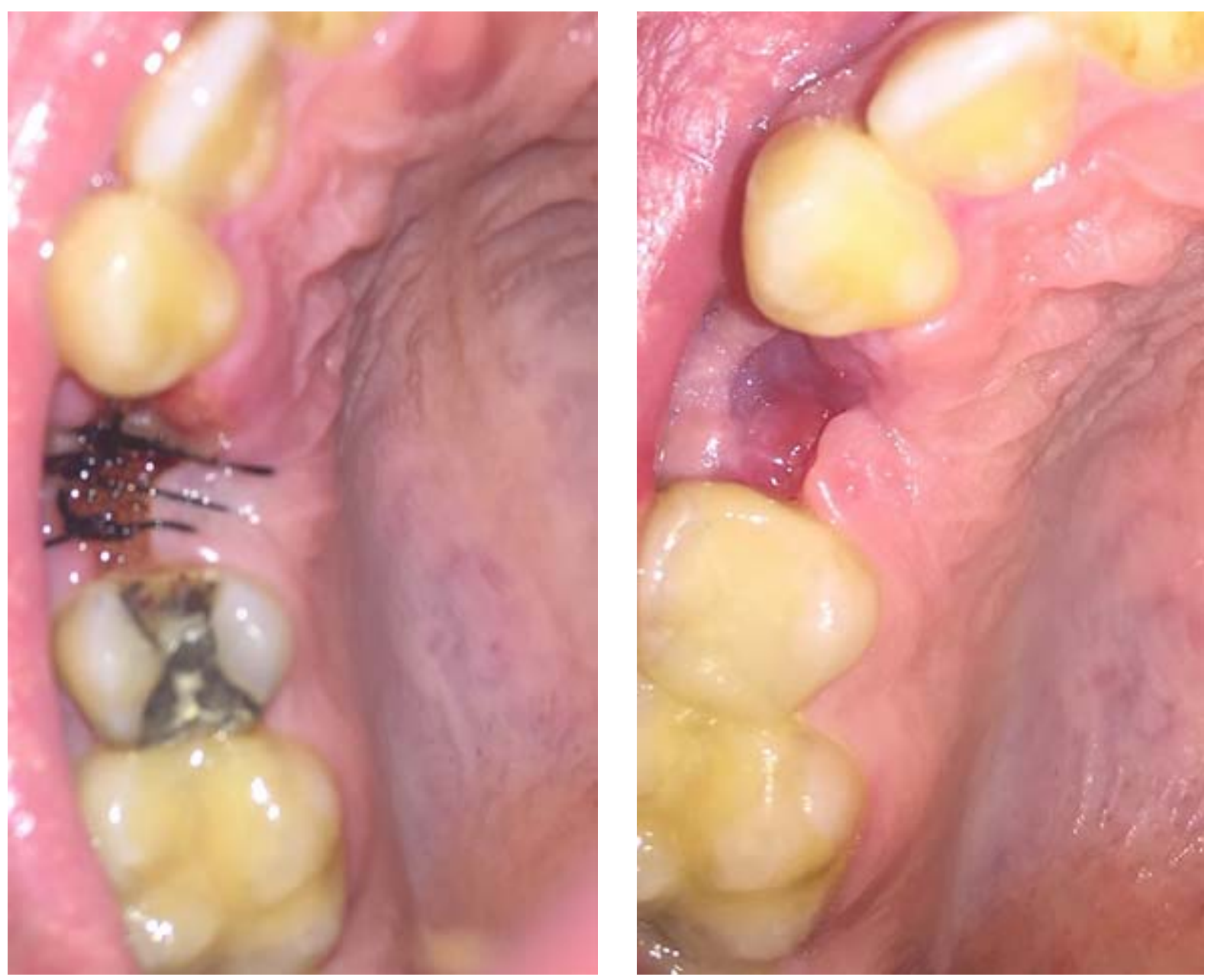

Figure 4. The Alveolar Socket: Seven Days (left) and Twenty-One Days (right) following Tooth Extraction

The patient completed his antimicrobial course treatment, and he gradually stopped taking analgesic medications and was pain-free. One week later, the stitches were removed, and the clinical examination in combination with a plain X-ray view confirmed that the healing was progressing well although there was a residual pus formation till day-15 and day-16 from the date of the tooth extraction. Finally, the patient consulted an oral and maxillofacial surgeon who confirmed that full healing and closure of the socket would ensue within one month. Based on this advice and his prior unpleasant experience following the tooth extraction, he decided to cancel his orthodontic management option and to go with dental implant procedures (Figure 5) for his extracted teeth in agreement with his maxillofacial surgeon. Possible risk factors for the development of dry socket in our presented case are not limited to the poor oral health, tooth overcrowding, pre-existing root canal fillings, gingival infection, traumatic and prolonged tooth extraction procedure, contamination of dentistry instruments, and vigorous mouth rinsing following the tooth extraction. 


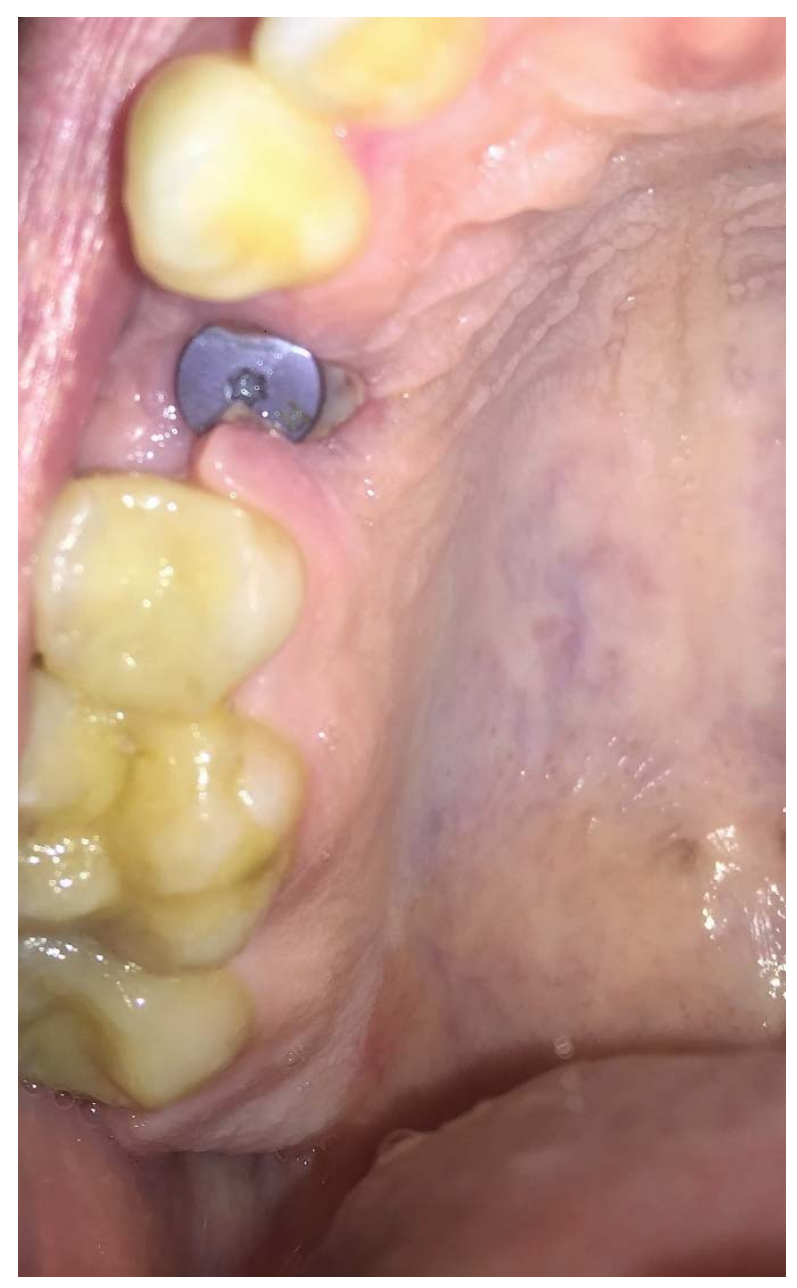

Figure 5. The Alveolar Socket: Successful Dental Implant

Our case presentation should prompt future research in the future, including case series and longitudinal analytics as well as eventual rigour pre-clinical and clinical trials, investigating the epidemiology, pathophysiology and risk factors, and diagnostics as well as therapeutics related to the development of dry socket and alveolar osteitis following complicated premolar teeth extraction surgeries. Aggregate and ecological studies based on correlations and regression models should be helpful in extrapolating an inference on the potential risk factors. Google Trends analytics in conjunction with machine learning algorithms can infer highly-reliable and real-time data on the topic (Al-Imam, 2019; Al-Imam et al., 2019). Therefore, it can be integrated into a peremptory warning system to anticipate any change in the geographic mapping and the digital epidemiology of rare medical conditions. The magnitude of benefit of real-time and predictive analytics can be of unique advantage when applied to epidemiological studies via sparing the workforce and financial resources.

\section{Acknowledgements}

The authors would like to acknowledge the supporting efforts of Dr Ahmed Al-Jawady who is a resident doctor of oral and maxillofacial surgery at the Iraqi Ministry of Health. Al-Jawady contributed to the medical management of the case reported in this study.

\section{References}

Abraham, M. A., Azari, A., Westcott, J., \& Stavropoulos, F. (2016). Dry Socket (Alveolar Osteitis, Fibrinolytic Osteitis). In Atlas of Emergency Medicine Procedures (pp. 373-374). Springer, New York, NY.

Abu-Mostafa, N. A., Alqahtani, A., Abu-Hasna, M., Alhokail, A., \& Aladsani, A. (2015). A randomized clinical trial compared the effect of intra-alveolar $0.2 \%$ Chlorohexidine bio-adhesive gel versus $0.12 \%$ Chlorohexidine rinse in reducing alveolar osteitis following molar teeth extractions. Medicina Oral Patologia Oral y Cirugia Bucal, 20(1), e82. 
Adams, R. J., Smart, P., \& Huff, A. S. (2017). Shades of grey: guidelines for working with the grey literature in systematic reviews for management and organizational studies. International Journal of Management Reviews, 19(4), 432-454.

Al-Imam A. (2019). Monitoring and Analysis of Novel Psychoactive Substances in Trends Databases, Surface Web and the Deep Web, with Special Interest and Geo-Mapping of the Middle East. info:eu-repo/semantics/masterThesis [dissertation on the Internet]. United Kingdom: University of Hertfordshire; 2017. https://doi.org/10.13140/RG.2.2.27636.24961

Al-Imam, A. (2019). A Gateway Towards Machine Learning: Predictive Analytics and Neural Networks in IBM-SPSS (SPSS v.24). https://doi.org/10.13140/RG.2.2.20885.99041/3

Al-Imam, A., \& AbdulMajeed, B. A. (2017). The NPS Phenomenon and the Deep Web: Internet Snapshots of the Darknet and Potentials of Data Mining. Global Journal of Health Science, 9(11), 86.

Al-Imam, A., Khalid, U., Al-Hadithi, N., \& Kaouche, D. (2019). Real-time Inferential Analytics Based on Online Databases of Trends: A Breakthrough Within the Discipline of Digital Epidemiology of Dentistry and Oral-Maxillofacial Surgery. Modern Applied Science, 13(2), 81-94.

Better Value Healthcare Ltd. CASP Checklists. Retrieved July 20, 2018, from https://casp-uk.net/casp-tools-checklists/

Biagioni, S. G. S., Goggi, S., \& Pardelli, G. (2017). Grey Literature Citations in the age of Digital Repositories and Open Access. Grey Journal (TGJ), 13(1).

Bienek, D. R., \& Filliben, J. J. (2016). Risk assessment and sensitivity meta-analysis of alveolar osteitis occurrence in oral contraceptive users. The Journal of the American Dental Association, 147(6), 394-404.

Blum, I. R. (2002). Contemporary views on dry socket (alveolar osteitis): a clinical appraisal of standardization, aetiopathogenesis and management: a critical review. International Journal of Oral and Maxillofacial Surgery, 31(3), 309-317.

Bosco, J. M. D., Oliveira, S. R. D., Bosco, Á. F., Schweitzer, C. M., \& Jardim Júnior, E. G. (2008). Influence of local tetracycline on the microbiota of alveolar osteitis in rats. Brazilian Dental Journal, 19(2), 119-123.

Bowe, D. D. C. (2011). The management of dry socket alveolar osteitis. Journal of Irish Dental Association, 57(6):305-10.

Cho, H., Lynham, A. J., \& Hsu, E. (2017). Postoperative interventions to reduce inflammatory complications after third molar surgery: review of the current evidence. Australian Dental Journal, 62(4), 412-419.

Cochrane. Cochrane Library. Retrieved July 28, 2018, from http://www.cochranelibrary.com/

Crawford, J. Y. (1896). Dry socket. Dent Cosmos, 38, 929.

Daly, B., Sharif, M. O., Newton, T., Jones, K., \& Worthington, H. V. (2012). Local interventions for the management of alveolar osteitis (dry socket). Cochrane Database of Systematic Reviews, (12).

de Wyels, S. V. C. (1998). Alveolar osteitis (dry socket) in a dog: a case report. Journal of Veterinary Dentistry, 15(2), 85-87.

Dubovina, D., Mihailović, B., Bukumirić, Z., Vlahović, Z., Miladinović, M., Miković, N., \& Lazić, Z. (2016). The use of hyaluronic and aminocaproic acid in the treatment of alveolar osteitis. Vojnosanitetski Pregled, 73(11), 1010-1015.

Elo, J. A., Sun, H. H. B., Dong, F., Tandon, R., \& Singh, H. M. (2016). Novel incision design and primary flap closure reduces the incidence of alveolar osteitis and infection in impacted mandibular third molar surgery. Oral Surgery, Oral Medicine, Oral Pathology and Oral Radiology, 122(2), 124-133.

Eshghpour, M., Ahrari, F., Najjarkar, N. T., \& Khajavi, M. A. (2015). Comparison of the effect of low level laser therapy with alvogyl on the management of alveolar osteitis. Medicina Oral Patologia Oral y Cirugia Bucal, 20(3), e386.

Eshghpour, M., Dastmalchi, P., Nekooei, A. H., \& Nejat, A. (2014). Effect of platelet-rich fibrin on frequency of alveolar osteitis following mandibular third molar surgery: a double-blinded randomized clinical trial. Journal of Oral and Maxillofacial Surgery, 72(8), 1463-1467.

Fernandes, G. J., \& Hatton, M. N. (2017). Prevention of Alveolar Osteitis: A Case Report and Review of Literature. Journal of the Michigan Dental Association, 82(1), 21-5. 
Freudenthal, N., Sternudd, M., Jansson, L., \& Wannfors, K. (2015). A double-blind randomized study evaluating the effect of intra-alveolar chlorhexidine gel on alveolar osteitis after removal of mandibular third molars. Journal of Oral and Maxillofacial Surgery, 73(4), 600-605.

Gašević, D., Dawson, S., \& Siemens, G. (2015). Let's not forget: Learning analytics are about learning. TechTrends, 59(1), 64-71.

Greenhalgh, T. (2014). How to read a paper: The basics of evidence-based medicine. John Wiley \& Sons.

Halabi, Diego, Escobar, Jose, Alvarado, Cyntia, Martinez, Nicolette, \& Muñoz, Carlos. (2018). Chlorhexidine for prevention of alveolar osteitis: a randomised clinical trial. Journal of Applied Oral Science, 26, e20170245.

Haraji, A., \& Rakhshan, V. (2015). Chlorhexidine gel and less difficult surgeries might reduce post-operative pain, controlling for dry socket, infection and analgesic consumption: a split-mouth controlled randomised clinical trial. Journal of Oral Rehabilitation, 42(3), 209-219.

Hay, S. I., George, D. B., Moyes, C. L., \& Brownstein, J. S. (2013). Big data opportunities for global infectious disease surveillance. PLoS medicine, 10(4), e1001413.

Hoffmann, T. C., Montori, V. M., \& Del Mar, C. (2014). The connection between evidence-based medicine and shared decision making. Journal of the American Medical Association, 312(13), 1295-1296.

Jesudasan, J. S., Wahab, P. A., \& Sekhar, M. M. (2015). Effectiveness of $0.2 \%$ chlorhexidine gel and a eugenol-based paste on postoperative alveolar osteitis in patients having third molars extracted: a randomised controlled clinical trial. British Journal of Oral and Maxillofacial Surgery, 53(9), 826-830.

Koyuncu, B. Ö., \& Çetingül, E. (2013). Short-term clinical outcomes of two different flap techniques in impacted mandibular third molar surgery. Oral Surgery, Oral Medicine, Oral Pathology and Oral Radiology, 116(3), e179-e184.

Krakowiak, P. A. (2011). Alveolar osteitis and osteomyelitis of the jaws. Oral and Maxillofacial Surgery Clinics, 23(3), 401-413.

Lent, B., Agrawal, R., \& Srikant, R. (1997). Discovering Trends in Text Databases. In Knowledge Discovery and Data Mining (Vol. 97, pp. 227-230).

Marcussen, K. B., Laulund, A. S., Jørgensen, H. L., \& Pinholt, E. M. (2016). A systematic review on effect of single-dose preoperative antibiotics at surgical osteotomy extraction of lower third molars. Journal of Oral and Maxillofacial Surgery, 74(4), 693-703.

Nasrabadi, N. M. (2007). Pattern recognition and machine learning. Journal of Electronic Imaging, 16(4), 049901.

Nuti, S. V., Wayda, B., Ranasinghe, I., Wang, S., Dreyer, R. P., Chen, S. I., \& Murugiah, K. (2014). The use of google trends in health care research: a systematic review. PloS one, 9(10), e109583.

Osunde, O. D., \& Bassey, G. O. (2015). Role of warm saline mouth rinse in prevention of alveolar osteitis: a randomized controlled trial. Editorial Board, 24(1).

Oxford Centre for Evidence-Based Medicine. OCEBM Levels of Evidence. Retrieved July 28, 2018, from https://www.cebm.net/2016/05/ocebm-levels-of-evidence/

Phillips, B., Ball, C., Badenoch, D., Straus, S., Haynes, B., \& Dawes, M. (2011). Oxford centre for evidence-based medicine levels of evidence (May 2001). BJU international, 107(5), 870.

Poulin, C., Thompson, P., \& Bryan, C. (2016). Public health surveillance: predictive analytics and big data. In Artificial Intelligence in Behavioral and Mental Health Care (pp. 205-230).

Rubio-Palau, J., Garcia-Linares, J., Hueto-Madrid, J. A., González-Lagunas, J., Raspall-Martin, G., \& Mareque-Bueno, J. (2015). Effect of intra-alveolar placement of $0.2 \%$ chlorhexidine bioadhesive gel on the incidence of alveolar osteitis following the extraction of mandibular third molars. A double-blind randomized clinical trial. Medicina Oral Patologia Oral y Cirugia Bucal, 20(1), e117.

Sanchez, F. R., Andrés, C. R., \& Calvo, I. A. (2017). Does chlorhexidine prevent alveolar osteitis after third molar extractions? Systematic review and meta-analysis. Journal of Oral and Maxillofacial Surgery, 75(5), 901-914.

Sharif, M. O., Dawoud, B. E. S., Tsichlaki, A., \& Yates, J. M. (2014). Interventions for the prevention of dry socket: an evidence-based update. British Dental Journal, 217(1), 27. 
Shea, B. J., Reeves, B. C., Wells, G., Thuku, M., Hamel, C., Moran, J., ... \& Henry, D. A. (2017). AMSTAR 2: a critical appraisal tool for systematic reviews that include randomised or non-randomised studies of healthcare interventions, or both. British Medical Journal, 358, j4008.

Steele, B., Chandler, J., \& Reddy, S. (2016). Real-time Analytics. In Algorithms for Data Science (pp. 381-401). Springer, Cham.

Taberner-Vallverdú, M., Sánchez-Garcés, M. Á., \& Gay-Escoda, C. (2017). Efficacy of different methods used for dry socket prevention and risk factor analysis: A systematic review. Medicina Oral Patologia Oral y Cirugia Bucal, 22(6), e750.

Tek, M., Akkas, I., Toptas, O., Ozan, F., Sener, I., \& Bereket, C. (2014). Effects of the topical hemostatic agent Ankaferd Blood Stopper on the incidence of alveolar osteitis after surgical removal of an impacted mandibular third molar. Nigerian Journal of Clinical Practice, 17(1), 75-80.

Teshome, A. (2017). The efficacy of chlorhexidine gel in the prevention of alveolar osteitis after mandibular third molar extraction: a systematic review and meta-analysis. BMC Oral Health, 17(1), 82.

Unsal, H., \& Erbasar, G. N. H. (2018). Evaluation of the effect of platelet-rich fibrin on the alveolar osteitis incidence and periodontal probing depth after extracting partially erupted mandibular third molars extraction. Nigerian Journal of Clinical Practice, 21(2), 201-205.

Wheeler, D. L., Barrett, T., Benson, D. A., Bryant, S. H., Canese, K., Chetvernin, V., ... \& Geer, L. Y. (2006). Database resources of the national center for biotechnology information. Nucleic Acids Research, 35(suppl_1), D5-D12.

Witten, I. H., Frank, E., Hall, M. A., \& Pal, C. J. (2016). Data Mining: Practical machine learning tools and techniques. Morgan Kaufmann.

Xue, P., Wang, J., Wu, B., Ma, Y., Wu, F., \& Hou, R. (2015). Efficacy of antibiotic prophylaxis on postoperative inflammatory complications in Chinese patients having impacted mandibular third molars removed: a split-mouth, double-blind, self-controlled, clinical trial. British Journal of Oral and Maxillofacial Surgery, 53(5), 416-420.

Yengopal, V., \& Mickenautsch, S. (2012). Chlorhexidine for the prevention of alveolar osteitis. International Journal of Oral and Maxillofacial Surgery, 41(10), 1253-1264.

Zhou, J., Hu, B., Liu, Y., Yang, Z., \& Song, J. (2017). The efficacy of intra-alveolar $0.2 \%$ chlorhexidine gel on alveolar osteitis: a meta-analysis. Oral Diseases, 23(5), 598-608.

\section{Copyrights}

Copyright for this article is retained by the author(s), with first publication rights granted to the journal.

This is an open-access article distributed under the terms and conditions of the Creative Commons Attribution license (http://creativecommons.org/licenses/by/4.0/). 